\title{
Assessment the Diversity of Circulating Natural Killer Cells Between Active Tuberculosis and Latent Tuberculosis Infection
}

Yu Zhou ( $\sim$ zhouyu201378@163.com )

Zhejiang Provincial People's Hospital, People's Hospital of Hangzhou Medical College

Haiping Lan

Affiliated Hospital of Hangzhou Normal University

Hanlu Shi

Zhejiang Chinese Medical University

Peihao Wu

Women's Hospital, School of Medicine, Zhejiang University

Yonglie Zhou

Zhejiang Provincial People's Hospital, People's Hospital of Hangzhou Medical College

\section{Research Article}

Keywords: ATB, LTBI, NK subsets, KLRG1, NKG2D

Posted Date: September 16th, 2021

DOl: https://doi.org/10.21203/rs.3.rs-861318/v1

License: (c) (1) This work is licensed under a Creative Commons Attribution 4.0 International License. Read Full License 


\section{Abstract}

Background: Tuberculosis (TB) is a leading global public health problem, but the mechanisms underlying the immunopathology of TB progression are not well understood. It is currently believed that Mycobacterium tuberculosis (Mtb) infection can modify NK cell phenotypic signatures. Hence, our study was designed to investigate the diversity of circulating NK cells between active TB and latent TB infection. Peripheral blood NK subsets, as well as their expression of activating and inhibitory membrane receptors in different TB-infected status were evaluated in the present study.

Results: Significant differences of NK phenotypes were observed in the ATB, LTBI and HC populations.

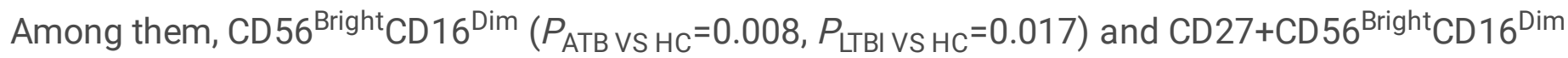
$\left(P_{\text {ATB V S HC }}=0.022, P_{\text {LTBI V HC }}=0.004\right)$ NK subsets were increased in TB-infected groups compared with HC group. On the contrary, the proportion of CD27 in NK cells $\left(P_{\text {ATB VS HC }}=0.036, P_{\text {LTBI VS HC }}=0.006\right)$ and CD56 ${ }^{\text {Dim }}$ CD 16+ NK subsets ( $P_{\text {ATB V }}$ HC $\left.=0.0001, P_{\text {LTBI VS HC }}=0.001\right)$ were diminished in TB-infected groups. Furthermore, the proportion of KLRG1 in NK cells $(P=0.036)$, as well as their subsets CD $56^{\text {Dim }}$ CD $16+N K$ $(P=0.046)$ and $\mathrm{CD} 27+\mathrm{NK}(P=0.027)$, were increased significantly in LTBI compared with the ATB group; while Mtb specific IFN- $\gamma+C D 56^{\text {Bright }^{2}}$ CD16 ${ }^{\text {Dim }}$ NK cells expressed higher KLRG1 in ATB than LTBI $(P=0.027)$. Within $\mathrm{CD} 56^{\text {Bright }} \mathrm{CD} 16^{\text {Dim }} \mathrm{NK}$ subsets, the percentage of KLRG1 was elevated in ATB patients compared with $\mathrm{HC}$ group ( $P=0.037)$. However, the expression of activating receptor NKG2D in NK and its subsets showed no significant between the three participant groups.

Conclusions: The present results demonstrated that the different TB infection states were coupled with the diversity of NK cell compartments, and the expression of KLRG1 in NK cells might be a specific phenotype to modulate the progression of TB from latent to active.

\section{Background}

Tuberculosis (TB) is the major cause of death from a single infectious disease. Despite the 2035 End TB Strategy has been committed by the World Health Organization, the number of TB infections has been declining slowly in recent years [1]. The latent TB infection (LTBI), that exhibits a persistent immune response to Mycobacterium tuberculosis (Mtb) antigen stimulation but without clinical evidence of active TB (ATB), affects approximately one-quarter of the world's population. Among them, $5 \%-10 \%$ will develop into ATB [2]. It is believed that this large population of LTBI at risk of activation will become the last bastion on the road of eradicating TB [3].

The lack of biomarkers to distinguish ATB from LTBI is an important challenge for TB control. Classically, $\mathrm{CD} 4+\mathrm{T}$ cells are believed to play an important role in antimycobacterial immune response. However, the interferon gamma release assay (IGRA) and tuberculin skin test (TST), which based on T cell immunity, cannot distinguish between ATB and LTBI [4]. A series of innate and specific immune responses are involved in anti-TB process. Recently, the role of natural killer (NK) cells in the progression of TB has been increasingly emphasized [5]. 
Several researches have reported that the expression of NK surface markers changes in the context of different diseases [6-8], but remain poorly understood in TB infection. NK cell diversity and function are tightly determined by a balance between signals from both activating and inhibitory membrane receptors [9]. Specifically, NK cell inhibitory receptors can be classified into four different families, of which KLRG1 belongs to the $\mathrm{C}$-type lectin like family. During persistent viral infections, KLRG1 is a marker of terminally differentiated CD8 + T cells, and the proportion of KLRG1 + T cells in peripheral blood increases [10-12], but its role in human TB infection is still elusive. In a mouse model of TB infection, $\mathrm{KLRG}^{-} /^{-}$mice significantly improved survival [13]; additionally, Hu et al. demonstrated that KLRG1 + CD4 + T cells were significantly increased in ATB patients compared with healthy controls, and suggested that KLRG1 blocking is a potential target for anti-TB immunotherapy [14]. However, in another study, the percentage of KLRG1 + CD 4 + T cells in ATB were the lowest, and the LTBI group was higher than ATB. Furthermore, they observed KLRG1 + CD4 + T cells increased after anti-TB treatment in ATB, which raised doubts about the immunotherapy that blocks KLRG1 [15]. Since Hu et al. did not conduct research on the LTBI group, the potential role of KLRG1 + CD4 + T cells in TB progression needs further study. Researches have shown that the interaction of KLRG1 and its ligand E-cadherin can increase the activation threshold of NK cells to inhibit cytotoxicity activity [16], and its expression correlates with functional distinctions of peripheral NK cells [17]. However, unlike T cells, the study of KLRG1 in NK cells is very rare, especially in TB infection.

Moreover, NK cells also express a large number of activating receptors. Among them, NKG2D is a well characterized activating receptor that recognizes several ligands poorly expressed on healthy cells but up regulated by several stressing stimuli, including cancer and infection [18]. By binding to its ligand, NKG2D can lead to cytokine secretion or cytotoxicity [19], increasing the bactericidal capacity of infected phagocytes. During TB infection, activated NK cells are important to kill extracellular Mtb. Nevertheless, as one of conserved activating receptors, NKG2D has not been widely studied in the course of TB.

In addition to the wide variability of receptors that regulate NK cell activity, there are growing evidence that NK cells can differentiate into several subpopulations with diverse functional profiles in different diseases. Classically, the subsets of NK cells were based on the expression of CD56 and CD16 [20]. The CD56 ${ }^{\text {dim }}$ CD 16 + NK subset representing the majority of circulating NK cells is mostly responsible for cytotoxicity, while the $\mathrm{CD} 56^{\text {bright }} \mathrm{CD} 16^{\mathrm{dim}}$ NK has more immunoregulatory function by producing abundant cytokines, such as IFN- $y$ [21]. To explore whether Mtb exposure and infection modifies NK cell phenotypic and functional signatures, Harris. et al found specific differences in CD56 ${ }^{\mathrm{dim}} \mathrm{NK}$ cell phenotype and function among QFT+, QFT-, and healthy individuals [22]. However, recent studies have reported that, the TNF-receptor family member CD27, can define phenotypically and functionally different human NK subsets as well [20,23]. Moreover, by constructing animal model, Esaulova et al. found that CD27 + NK cells had a protective effect on the latent period of TB [24].

Inspired by evidence above, our study was designed to simultaneously explore the diversity of NK activating and inhibitory membrane receptors in different TB infection status with an emphasis on NKG2D and KLRG1expression. Meanwhile, whether Mtb infection modifies specific NK subtypes remain 
incompletely studied, the different distribution of NK subsets between ATB and LTBI were also investigated in this study. Remarkably, we reported here the expression of KLRG1 in NK cells might act as an important immunomodulatory molecule in the progression of TB, and its magnitude is significant in maintaining the immune control of TB latency.

\section{Methods}

\section{Subjects and sample collection}

This was a prospective study performed from 2020 to 2021 at Zhejiang Provincial People's Hospital. All participants were classified into three categories: ATB, LTBI, and healthy controls (HC). The ATB group included symptomatic patients with positive sputum smears and/or Mtb-specific PCR and/or cultures for Mtb. Asymptomatic healthy individuals from close contacts of ATB patients were classified as LTBI if diagnosed positive in IGRA, otherwise as $\mathrm{HC}$ cohort. Clinical and demographic data from participants were obtained by direct clinical interview and review of their medical records. All participants were seronegative for HIV, and ATB patients were enrolled before the initiation of anti-TB therapy. Individuals with primary or acquired immunosuppression were excluded. This study has been approved by the ethics committee of Zhejiang Provincial People's Hospital, Affiliated People's Hospital, Hangzhou Medical College, Hangzhou, Zhejiang, China. Informed consent was obtained from all participants.

\section{PBMC isolation and Stimulation}

Samples of peripheral blood were collected from all participants in sodium heparin tubes. Peripheral blood mononuclear cells (PBMCs) were isolated via Ficoll-Hypaque density gradient centrifugation. Afterwards, PBMCs were incubated into 96-well plates at a final concentration of $2 \times$ $10^{6} / \mathrm{mL}$ in complete medium (RPMI 1640 supplemented with $10 \%$ heat-inactivated fetal calf serum, 100 $\mathrm{U} / \mathrm{ml}$ penicillin, $100 \mu \mathrm{g} / \mathrm{ml}$ streptomycin, and 2mM L-glutamine). ESAT-6 \& CFP10 peptide pools (Oxford Immunotec, Oxford, England) were used as Mtb specific antigens; Phytohemagglutinin (PHA) was used as positive control (Oxford Immunotec, Oxford, England), and medium only was used as negative control. All Cells were also performed with anti-CD28 and anti-CD49d co-stimulatory antibodies (BD Pharmingen; final concentration $2 \mu \mathrm{g} / \mathrm{mL}$ each). After that, PBMCs were left in the incubator at $37^{\circ} \mathrm{C}$ and $5 \% \mathrm{CO}_{2}$ overnight. Brefeldin A (Sigma-Aldrich; final concentration $5 \mu \mathrm{g} / \mathrm{ml}$ ) was added at the final $5 \mathrm{~h}$ of incubation.

\section{Flow cytometric analysis}

After stimulation, PBMCs were collected and subsequently, the following surface markers were added to the cell suspensions in different tubes: anti-CD3-APC-Cy7, anti-CD56-BV510, anti-CD16-BB700, anti-CD27PE-Cy7, anti-KLRG1-APC, and anti-NKG2D-PE. All antibodies were purchased from BD Pharmingen. 
Representative flow cytometry gating strategies were showed in Fig. 1a and the additional file 1. Gated on CD3-CD 56+ cells, the different NK subsets were gated as the following criteria: (1) CD56 ${ }^{\mathrm{Dim}} \mathrm{CD} 16+$ and CD56 ${ }^{\text {Bright }}$ CD16 ${ }^{\text {Dim }}$ based on the expression of CD56 and CD16; (2) NK cell subpopulations defined by the expression of CD27. For intracellular staining, the cells were collected after surface staining. After fixing and permeabilizing, cells were further stained for intracellular markers (anti-IFN- $y-B V 421$ ). Finally, Samples were subjected to flow cytometric analysis on a BD FACS Canto II flow cytometer. Fluorescence Minus One (FMO) was performed in some immune indexes as the basis for door gating. Data analysis was conducted through FlowJo version 10.7.1 software.

\section{QuantiFERON (QFT) Assay}

QFT was employed as IGRA to evaluate the TB infection status of all participants. The QFT detection was performed according to the manufacturer's instruction (Wantai, Beijing, China). Briefly, blood samples were obtained and divided into three tubes: (1) a negative control tube (Nil tube), (2) a Mtb specific antigen stimulation (Test tube), and (3) a positive control tube containing PHA (PHA tube). After incubation at $37^{\circ} \mathrm{C}$ for about 22 hours, the supernatants were collected and IFN- $\gamma$ was quantitated by automated immunoassays Wantai Caris 200. The QFT results were judged according to the manufacturer's instruction.

\section{Statistical Analysis}

In order to describe the characteristics of study population, percentiles and the mean \pm SD were used. Differences of clinical and laboratory data between groups were analyzed using Student's $t$ test or Wilcoxon rank-sum test where appropriate. The Fisher's exact test or chi-square $(\chi 2)$ test was used for categorical data. Statistical analyses were performed using GraphPad Prism 8.4.2 and SPSS 22.0. $P<$ 0.05 was considered statistically significant.

\section{Results}

\section{Participant Characteristics}

There were 20 ATB, 13 LTBI and 14 HC participants enrolled for immunological analysis. All three groups were similar in age and sex characteristics. The main characteristics of ATB patients were shown in Table 1. The mean age of ATB group was 52.71 years old, and $65 \%$ of participants were male. More than half of ATB patients were confirmed by culture and/or molecular methods; $10 \%$ of them were QFT negative. Pulmonary tuberculosis (PTB) is the major type of ATB patients. Meanwhile, peripheral blood leukocytes, neutrophils and lymphocytes were detected in ATB cohort. Compared with the normal reference interval of healthy people, leukocytes and neutrophils were increased in $15 \%$ and $10 \%$ of ATB patients respectively, while lymphocytes were reduced in $35 \%$ of ATB. 


\section{Table 1}

Clinical and laboratory characteristics of ATB patients

\begin{tabular}{|c|c|}
\hline Characteristics & $\mathrm{N}=\mathbf{2 0}$ \\
\hline Age (years) Mean $\pm S D$ & $52.71 \pm 22.06$ \\
\hline Male Sex (n, \%) & $13(65 \%)$ \\
\hline Smear Positive (n, \%) & $3(15 \%)$ \\
\hline Culture (n, \%) & $11(55 \%)$ \\
\hline $\operatorname{PCR}(n, \%)$ & $11(55 \%)$ \\
\hline IGRA (n, \%) & $18(90 \%)$ \\
\hline PTB $(n, \%)$ & $15(75 \%)$ \\
\hline EPTB $(n, \%)$ & $5(25 \%)$ \\
\hline Leukocytes $\left(\times 10^{9} / \mathrm{L}\right)$ & $6.70 \pm 2.26$ \\
\hline Neutrophils (\%) & $62.87 \pm 12.77$ \\
\hline Lymphocytes (\%) & $25.55 \pm 11.34$ \\
\hline
\end{tabular}

\section{NK subsets within study groups}

Using flow cytometry, circulating CD3+CD56+ NKT, CD3-CD56+ NK and its subsets (based on CD 56 and CD16 expression) were analyzed among ATB, LTBI and HC cohorts (Fig.1). Compared with HC group, NKT cells were significantly increased in the ATB patients $(P=0.02)$. Although there was no statistical difference in the percentage of CD3-CD56+ NK cells among three cohorts, statistical significance was observed in NK subgroup analysis. Among them, CD56 ${ }^{\text {Bright }}$ CD $16^{\text {Dim }}$ NK cells were significantly higher in TB-infected groups (ATB and LTBI; $P_{\text {ATB VS HC }}=0.008, P_{\text {LTBI VS HC }}=0.017$ ).

\section{Expression of surface activating and inhibitory receptors in peripheral NK subpopulations.}

As the function of NK cells are governed by a variety of activating and inhibitory receptors, we simultaneously evaluated the expression of representative activating and inhibitory NK cell receptors, NKG2D and KLRG1, to determine if the progression of TB is related to the imbalanced receptor expression of NK cells (Fig.2 a-b). Interestingly, LTBI populations tended to have higher KLRG1 expression. The 
proportion of KLRG1 in NK cells $(P=0.036)$, as well as their subsets $C D 56^{\mathrm{Dim}} \mathrm{CD} 16+\mathrm{NK}(P=0.046)$, were increased significantly in LTBI compared with the ATB group. Furthermore, within CD $56^{\text {Bright }}$ CD $16^{\text {Dim }} \mathrm{NK}$ subsets, the percentage of KLRG1 was elevated in ATB patients compared with HC group $(P=0.037)$. However, their expression of the activating receptor NKG2D, showed no significant difference between the three participant groups. We also compared the expression of surface immune indexes in NKT cells, but the frequency of NKG2D and KLRG1 in NKT failed to reach significance in the participant cohorts (see Additional file 2).

\section{Cytokine secretion capacity of NK subsets expressing KLRG1 or NKG2D}

As a major cytokine involved in the TB immune response, IFN- $\gamma$ was found to be significantly increased in NKT and NK cell subsets of ATB patients compared with LTBI cohort (Fig. $2 \mathrm{c}$ and Additional file 2). When comparing the IFN-y secretion capacity of NK subsets expressing KLRG1 or NKG2D receptor, we found IFN- $\gamma$ was highly secreted on KLRG1 - and NKG2D+ NK compared with KLRG1+ and NKG2D- NK cells (Fig. 3). Moreover, IFN-y levels in both positive or negative expression of KLRG1 and NKG2D on CD 56 ${ }^{\text {Dim }}$ CD 16+NK cells were capable of distinguishing ATB from LTBI.

To further delineate the relationship between surface immune indicators and IFN- $\gamma$ secretion in TBinfected population, we analyzed the expression of KLRG1 and NKG2D in Mtb specific IFN-y positive NK cells between ATB and LTBI cohorts. Remarkably, IFN- $\gamma+C D 56^{\text {Bright }}$ CD $16^{\text {Dim }}$ NK cells showed higher KLRG1 expression in ATB than LTBI ( $P=0.027$, Fig. 4 a). But beyond that, no other significant differences were observed between ATB and LTBI individuals.

\section{NK subsets defined by CD27}

Because it has been reported that CD27 is an additional important marker for dividing NK subgroups [20], NK subsets defined by CD27 were also analyzed to explore their potential value in human immunity against TB. The proportion of CD27 in NK cells ( $P_{\text {ATB VS HC }}=0.036, P_{\text {LTBI VS HC }}=0.006$; Fig. 5 a) and CD56 ${ }^{\text {Dim }}$ CD16+ NK subsets $\left(P_{\text {ATB VS HC }}=0.0001, P_{\text {LTBI VS HC }}=0.001 ;\right.$ Fig. 5 b) were diminished in TB-infected groups; on the contrary, CD27+CD56 ${ }^{\text {Bright }} \mathrm{CD} 16^{\mathrm{Dim}} \mathrm{NK}$ cells were increased in ATB and LTBI groups when compared with $\mathrm{HC}$ group $\left(P_{\mathrm{ATB}}\right.$ VS HC $\left.=0.022, P_{\text {LTBI VS HC }}=0.004\right)$. To better demonstrate the special value of CD27 defined NK cells in TB infection, the expression of NK cell surface receptors and the production of IFN- $\gamma+$ between CD27- and CD27+ NK cells were measured as well. Similarly, the proportion of KLRG1 in CD27+NK cells was significantly elevated in LTBI than ATB ( $P_{\text {ATB Vs LTBI }}=0.027$, Fig. 6 a). Although KLRG1 expression in CD27+ and CD27- NK cells were not statistically different, CD27+NK subsets expressed higher NKG2D receptor than CD27- subsets (Fig. 6 b). In addition, CD27+NK cells were more likely to produce IFN- $y$ than their CD27- counterparts, but this failed to reach a statistical difference (see Additional file 3). 


\section{Discussion}

NK cells have a pivotal role in the innate immune response against viruses and cancer. However, studies on the role of NK in anti-TB immunity are limited. In order to investigate if Mtb infection modifies NK cell phenotypic signatures, we estimated circulating NK subsets, as well as their expression of KLRG1 and NKG2D in different TB-infected status. Significant different of NK phenotypes were observed among the ATB, LTBI and HC populations. Compared with $\mathrm{HC}$ group, $\mathrm{CD} 56^{\text {Bright }} \mathrm{CD} 16^{\mathrm{Dim}}$ and $\mathrm{CD} 27+$ CD56 ${ }^{\text {Bright }}$ CD $16^{\text {Dim }}$ NK were increased in TB-infected groups. Furthermore, LTBI individuals showed significantly increased KLRG1 + NK cells than ATB patients, while Mtb specific IFN- $\gamma+$ CD $56^{\text {Bright }}$ CD $16^{\text {Dim }}$ NK cells expressed higher KLRG1 in ATB than LTBI. Our study adds novel information on KLRG1 expressed NK cells, which might be a specific phenotype to modulate the progression of TB from latent to active.

Encouraged by the hypothesis that NK cell function and subsets in the blood can inform Mtb infection status [25], circulating NK subsets from ATB, LTBI and HC cohorts were analyzed by flow cytometry. In line with other evidence $[22,25]$, the frequency of CD3 - CD56 + NK cells were similar in the three participant groups. Generally speaking, NK functions can be preferentially assigned to two major NK subsets, with the $\mathrm{CD} 56^{\mathrm{Dim}} \mathrm{CD} 16$ + subset being intrinsically highly cytotoxic and the CD $56^{\text {Bright }} \mathrm{CD} 16^{\mathrm{Dim}}$ subset being more efficient to produce cytokines, mainly IFN- $[26]$. By releasing IFN- - , the CD56 ${ }^{\text {Bright }}$ CD 16 ${ }^{\text {Dim }}$ NK cells are assumed to provide protective mechanisms in LTBI subjects [27]. However, we observed that the percentage of $\mathrm{CD} 56^{\text {Bright }} \mathrm{CD} 16^{\mathrm{Dim}} \mathrm{NK}$ cells were significantly increased in both ATB and LTBI groups. Although NK functions tend to be exhausted during the long-term progression of TB $[28,29]$, the abundance of $C D 56^{\text {Bright }} \mathrm{CD} 16^{\mathrm{Dim}}$ subsets in TB-infected groups probably reflects their important role in the early Mtb responsive accessory cells.

NKT, along with NK, is known as the bridge between innate and adaptive immune response. The role of NKT cells in the context of Mtb infection is still controversial. Consistent with the results of Pandey et al. [30], we found that NKT cells were elevated in ATB patients compared with HC individuals, which further indicated the importance of innate immunity to against TB.

Given that NK cell surface marker redistribution has been described in the setting of some infections [31, 32], typical activating and inhibitory receptors expressed by NK subsets, and their ability to secrete IFN- $y$ were studied in each of the three participant groups. KLRG1 is widely used as a lymphocyte differentiation marker in both humans and mice but the physiological role of KLRG1 in TB is still unclear. Our results revealed that KLRG1 expressing NK cells, as well as their subsets CD $56^{\mathrm{Dim}} \mathrm{CD} 16+$ and CD27 + NK cells were reduced in ATB patients compared with LTBI individuals, which was similar to another report on the role of KLRG1 in human CD4 + T cells [15]. There is undoubtedly better immune control in $\mathrm{LTBI}$, our results indicated that KLRG1 + NK was an important component affecting the balance between host immunity and Mtb to keep TB latent. Whereas Hu et al. reported that KLTG1 expression in CD4 + T cells was significantly increased in ATB patients compared with $\mathrm{HC}$ subjects [14], the difference in research objects could partly explain the above discrepancy. Nevertheless, when compared with HC 
group, the percentage of KLRG1 + CD56 ${ }^{\text {Bright }}$ CD $16^{\text {Dim }}$ NK subsets was also elevated in ATB patients. In the above study, KLRG1 + CD $4+T$ cells tended to produce more IF- $y$ than KLRG1 - counterparts in ATB patients. However, IFN- $\gamma$ was found highly secreted on KLRG1 - NK cells compared with KLRG1 + counterparts in the present study. Through literature search, little information is currently available about the expression of KLRG1 in NK cells, especially about its role in NK subset function during TB infection. Nevertheless, similar results have been confirmed in other studies on HCMV infection [33]. In addition,

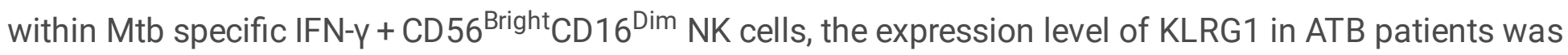
higher than that in LTBI population, which reflected the trend of NK cell function depletion during TB progression. In brief, the above results indicated that KLRG1 as an inhibitory receptor of NK cells plays an important role in anti-TB immunity, and circulating KLRG1 + NK cells may reflect the difference of host immune status between ATB and LTBI. Future studies evaluating the role of KLRG1 + NK cells during the course of TB are warranted.

Interestingly, the activating receptor NKG2D did not significantly affect NK diversity during TB infection, with no significant changes were observed in the participant groups. Normally, NKG2D are involved in the NK cell mediated lysis of human Mtb infected monocytes by enhancing the production of perforin and granulysin [34]; we found that NK cells expressing NKG2D also had a stronger IFN- $y$ secretion capacity compared with NKG2D - subjects.

The CD27 marker is a member of the TNF-receptor superfamily, and its low expression in IFN- $\gamma+C D 4+T$ cells has been proposed as an active TB marker $[35,36]$. Although the CD27 expression varies on murine NK cells [37], little is known about the expression of this receptor in human NK cells. We here showed that CD27 was expressed differently in NK subsets based on CD56 and CD16 typing. Generally, the expression of CD27 is associated with immunoregulatory and cytokine producing [23]. Consistent with the above statement, CD27 was found mainly expressed in CD56 ${ }^{\text {Bright }}$ CD $16^{\text {Dim }}$ NK cells and up regulated in TBinfected groups, which explained the response of ATB and LTBI individuals to secrete IF-y against Mtb. Furthermore, the phenomenon that CD27 + NK subsets expressed higher NKG2D than CD27 - subsets further demonstrated our conclusion above that NKG2D + NK cells tended to secrete more IFN-y than negative cells.

This study also had some limitations. First of all, it was a single center study, and the statistical power of analysis may be insufficient due to the limited sample size. Secondly, other activating and inhibitory receptors, such as NKp46 and the KIR family; as well as NK function markers, such as CD107a and granzyme B, were not systematically evaluated in the presents study. Finally, the fluctuations of NK subsets during TB treatment were not monitored as well. Further researches are needed to fill these gaps.

\section{Conclusions}

We demonstrated here that the different TB infection states were coupled with diversity of NK cell compartments, and KLRG1 + NK cells appeared to be key factors associated with maintaining TB latency. These cells may prove to be useful for targeted immune interventions for disease detection and control. 
Our research can lead to a better understanding of NK immune response during Mtb infection, which could be instrumental in the control of TB progression.

\section{Abbreviations}

TB, Tuberculosis; LTBI, The latent tuberculosis infection; Mtb, Mycobacterium tuberculosis; ATB, Active tuberculosis; IGRA, Interferon gamma release assay; TST, tuberculin skin test; NK, natural killer; PTB, Pulmonary tuberculosis; EPTB, extrapulmonary tuberculosis; HC, healthy controls; PBMCs, Peripheral blood mononuclear cells; FMO, Fluorescence Minus One; QFT, QuantiFERON; PHA, Phytohemagglutinin.

\section{Declarations}

\section{Acknowledgements}

We would like to thank the clinical and laboratory staff of the Zhejiang Provincial People's Hospital, Hangzhou, China, for facilitating the collection and measurement of the samples.

\section{Authors' contributions}

HL was responsible for recruitment of patients and samples collection. HS and YZ were in charge of laboratory procedures. HS and PW were involved in data analysis and interpretation. $\mathrm{YZ}$ and $\mathrm{HL}$ designed the study and took a leading role in writing the manuscript. All authors drafted the article and approved the final manuscript.

\section{Funding}

This work was supported by the National Natural Science Foundation of China (81902132). The funders had no role in study design, data collection and analysis, decision to publish, or preparation of the manuscript.

\section{Availability of data and materials}

All data supporting the conclusions of this article are presented within the article and its additional files 1 , 2 and 3.

\section{Declarations}

All procedures performed in the study were approved by the ethics committee of Zhejiang Provincial People's Hospital, Affiliated People's Hospital, Hangzhou Medical College, Hangzhou, Zhejiang, China 
(2019KY224). Informed written consent was obtained from the parents or legal guardian of the patient before inclusion in the study.

\section{Consent for publication}

Not applicable.

\section{Competing interests}

The authors declare that they have no competing interests.

\section{Author details}

${ }^{1}$ Department of Clinical Laboratory, Zhejiang Provincial People's Hospital, People's Hospital of Hangzhou Medical College, Zhejiang 310014, Hangzhou, China; ${ }^{2}$ Department of Tuberculosis, Affiliated Hospital of Hangzhou Normal University, Zhejiang 310015, Hangzhou, China; ${ }^{3}$ School of Medical Technology and Information Engineering, Zhejiang Chinese Medical University, Zhejiang 310053, Hangzhou, China; ${ }^{4}$ Department of Clinical Laboratory, School of Medicine, Women's Hospital, Zhejiang University, Zhejiang 310006, Hangzhou, China.

\section{References}

1. World Health Organization (WHO). Global Tuberculosis Report 2020. www.who.inttbpublicationsglobal_reporten. Date last updated 2020.pdf.

2. Carranza C, Pedraza-Sanchez S, de Oyarzabal-Mendez E, Torres M: Diagnosis for Latent Tuberculosis Infection: New Alternatives. Frontiers in immunology 2020, 11:2006.

3. LoBue PA, Mermin JH: Latent tuberculosis infection: the final frontier of tuberculosis elimination in the USA. The Lancet Infectious Diseases 2017, 17(10):e327-e333.

4. Abubakar I, Lalvani A, Southern J, Sitch A, Jackson C, Onyimadu O, Lipman M, Deeks JJ, Griffiths C, Bothamley $\mathrm{G}$ et al: Two interferon gamma release assays for predicting active tuberculosis: the UK PREDICT TB prognostic test study. Health Technol Assess 2018, 22(56):1-96.

5. Liu CH, Liu H, Ge B: Innate immunity in tuberculosis: host defense vs pathogen evasion. Cell Mol Immunol 2017, 14(12):963-975.

6. Hasan MS, Ryan PL, Bergmeier LA, Fortune F: Circulating NK cells and their subsets in Behcet's disease. Clin Exp Immunol 2017, 188(2):311-322.

7. Soares LS, Espindola MS, Zambuzi FA, Galvao-Lima LJ, Cacemiro MC, Soares MR, Santana BA, Calado RT, Bollela VR, Frantz FG: Immunosenescence in chronic HIV infected patients impairs essential functions of their natural killer cells. Int Immunopharmacol 2020, 84:106568. 
8. Zhang Y, Zhao Y, Si W, Yang B, Lin M, Zheng J, Cui L: Increased Peripheral NKG2A-NKG2D + CD3CD16 + CD56dim NK Cell Subset Was Positively Correlated with Antiphospholipid Antibodies in Patients of Obstetric Antiphospholipid Syndrome. Immunological Investigations 2020:1-14.

9. Horowitz A, Strauss-Albee DM, Leipold M, Kubo J, Nemat-Gorgani N, Dogan OC, Dekker CL, Mackey S, Maecker H, Swan GE et al: Genetic and environmental determinants of human NK cell diversity revealed by mass cytometry. Science translational medicine 2013, 5(208):208ra145.

10. Wang S, Zhang Q, Hui H, Agrawal K, Karris MAY, Rana TM: An atlas of immune cell exhaustion in HIVinfected individuals revealed by single-cell transcriptomics. Emerg Microbes Infect 2020, 9(1):23332347.

11. Hoji A, Popescu ID, Pipeling MR, Shah PD, Winters SA, McDyer JF: Early KLRG1(+) but Not CD57(+)CD8(+) T Cells in Primary Cytomegalovirus Infection Predict Effector Function and Viral Control. Journal of immunology 2019, 203(8):2063-2075.

12. Wijaya RS, Read SA, Schibeci S, Eslam M, Azardaryany MK, El-Khobar K, van der Poorten D, Lin R, Yuen L, Lam V et al: KLRG1 + natural killer cells exert a novel antifibrotic function in chronic hepatitis B. J Hepatol 2019, 71(2):252-264.

13. Cyktor JC, Carruthers B, Stromberg P, Flano E, Pircher H, Turner J: Killer cell lectin-like receptor G1 deficiency significantly enhances survival after Mycobacterium tuberculosis infection. Infect Immun 2013, 81(4):1090-1099.

14. Hu Z, Zhao HM, Li CL, Liu XH, Barkan D, Lowrie DB, Lu SH, Fan XY: The Role of KLRG1 in Human CD4 + T-Cell Immunity Against Tuberculosis. J Infect Dis 2018, 217(9):1491-1503.

15. Tonby K, Mortensen R, Ruhwald M, Dyrhol-Riise AM, Jenum S: KLRG1-Expressing CD4 T Cells Are Reduced in Tuberculosis Patients Compared to Healthy Mycobacterium tuberculosis-Infected Subjects, but Increase With Treatment. J Infect Dis 2019, 220(1):174-176.

16. Li Y, Hofmann M, Wang Q, Teng L, Chlewicki LK, Pircher H, Mariuzza RA: Structure of natural killer cell receptor KLRG1 bound to E-cadherin reveals basis for $\mathrm{MHC}$-independent missing self recognition. Immunity 2009, 31(1):35-46.

17. Huntington ND, Tabarias H, Fairfax K, Brady J, Hayakawa Y, Degli-Esposti MA, Smyth MJ, Tarlinton DM, Nutt SL: NK cell maturation and peripheral homeostasis is associated with KLRG1 up-regulation. Journal of immunology 2007, 178(8):4764-4770.

18. McCarthy MT, Lin D, Soga T, Adam J, O'Callaghan CA: Inosine pranobex enhances human NK cell cytotoxicity by inducing metabolic activation and NKG2D ligand expression. Eur J Immuno/ 2020, 50(1):130-137.

19. Andre P, Castriconi R, Espeli M, Anfossi N, Juarez T, Hue S, Conway H, Romagne F, Dondero A, Nanni $M$ et al: Comparative analysis of human NK cell activation induced by NKG2D and natural cytotoxicity receptors. Eur J Immunol 2004, 34(4):961-971.

20. Vossen MT, Matmati M, Hertoghs KM, Baars PA, Gent MR, Leclercq G, Hamann J, Kuijpers TW, van Lier RA: CD27 defines phenotypically and functionally different human NK cell subsets. Journal of immunology 2008, 180(6):3739-3745. 
21. Naujoks W, Quandt D, Hauffe A, Kielstein H, Bahr I, Spielmann J: Characterization of Surface Receptor Expression and Cytotoxicity of Human NK Cells and NK Cell Subsets in Overweight and Obese Humans. Frontiers in immunology 2020, 11:573200.

22. Harris LD, Khayumbi J, Ongalo J, Sasser LE, Tonui J, Campbell A, Odhiambo FH, Ouma SG, Alter G, Gandhi NR et al: Distinct Human NK Cell Phenotypes and Functional Responses to Mycobacterium tuberculosis in Adults From TB Endemic and Non-endemic Regions. Front Cell Infect Microbiol 2020, 10:120.

23. Gao M, Yang Y, Li D, Ming B, Chen H, Sun Y, Xiao Y, Lai L, Zou H, Xu Y et al: CD27 natural killer cell subsets play different roles during the pre-onset stage of experimental autoimmune encephalomyelitis. Innate Immun 2016, 22(6):395-404.

24. Esaulova E, Das S, Singh DK, Choreno-Parra JA, Swain A, Arthur L, Rangel-Moreno J, Ahmed M, Singh B, Gupta A et al: The immune landscape in tuberculosis reveals populations linked to disease and latency. Cell host \& microbe 2021, 29(2):165-178 e168.

25. Garand M, Goodier M, Owolabi O, Donkor S, Kampmann B, Sutherland JS: Functional and Phenotypic Changes of Natural Killer Cells in Whole Blood during Mycobacterium tuberculosis Infection and Disease. Frontiers in immunology 2018, 9:257.

26. Cooper MA, Fehniger TA, Caligiuri MA: The biology of human natural killer-cell subsets. Trends in immunology 2001, 22(11):633-640.

27. Barcelos W, Sathler-Avelar R, Martins-Filho OA, Carvalho BN, Guimarães TM, Miranda SS, Andrade HM, Oliveira MH, Toledo VP: Natural killer cell subpopulations in putative resistant individuals and patients with active Mycobacterium tuberculosis infection. Scandinavian journal of immunology 2008, 68(1):92-102.

28. Wang F, Hou H, Wu S, Tang Q, Huang M, Yin B, Huang J, Liu W, Mao L, Lu Y et al: Tim-3 pathway affects NK cell impairment in patients with active tuberculosis. Cytokine 2015, 76(2):270-279.

29. Hassan SS, Akram M, King EC, Dockrell HM, Cliff JM: PD-1, PD-L1 and PD-L2 Gene Expression on TCells and Natural Killer Cells Declines in Conjunction with a Reduction in PD-1 Protein during the Intensive Phase of Tuberculosis Treatment. PloS one 2015, 10(9):e0137646.

30. Pandey P, Bhatnagar AK, Mohan A, Sachdeva KS, Vajpayee M, Das BK, Samantaray JC, Guleria R, Singh UB: Insights in tuberculosis immunology: Role of NKT and T regulatory cells. Int $J$ Mycobacteriol 2019, 8(4):333-340.

31. Njiomegnie GF, Read SA, Fewings N, George J, McKay F, Ahlenstiel G: Immunomodulation of the Natural Killer Cell Phenotype and Response during HCV Infection. J Clin Med 2020, 9(4).

32. Maucourant C, Filipovic I, Ponzetta A, Aleman S, Cornillet M, Hertwig L, Strunz B, Lentini A, Reinius B, Brownlie D et al: Natural killer cell immunotypes related to COVID-19 disease severity. Science immunology 2020, 5(50).

33. Wang JM, Cheng YQ, Shi L, Ying RS, Wu XY, Li GY, Moorman JP, Yao ZQ: KLRG1 negatively regulates natural killer cell functions through the Akt pathway in individuals with chronic hepatitis $C$ virus infection. J Virol 2013, 87(21):11626-11636. 
34. Lu CC, Wu TS, Hsu YJ, Chang CJ, Lin CS, Chia JH, Wu TL, Huang TT, Martel J, Ojcius DM et al: NK cells kill mycobacteria directly by releasing perforin and granulysin. J Leukoc Bio/ 2014, 96(6):11191129.

35. Latorre I, Fernandez-Sanmartin MA, Muriel-Moreno B, Villar-Hernandez R, Vila S, Souza-Galvao ML, Stojanovic Z, Jimenez-Fuentes MA, Centeno C, Ruiz-Manzano J et al: Study of CD27 and CCR4 Markers on Specific CD4(+) T-Cells as Immune Tools for Active and Latent Tuberculosis Management. Frontiers in immunology 2018, 9:3094.

36. Xu F, Zhang H, Si X, Chen J, Chen Y, Cui X, Qin Y: Assessment of CD27 expression on T-cells as a diagnostic and therapeutic tool for patients with smear-negative pulmonary tuberculosis. $B M C$ Immunol 2021, 22(1):41.

37. Hayakawa Y, Smyth MJ: CD27 dissects mature NK cells into two subsets with distinct responsiveness and migratory capacity. Journal of immunology 2006, 176(3):1517-1524.

\section{Figures}

(a)

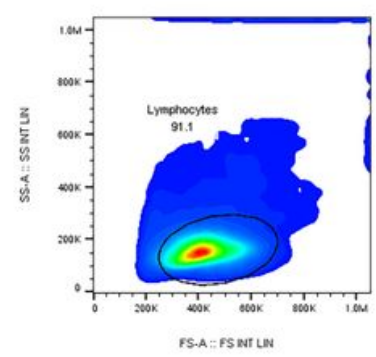

(b)

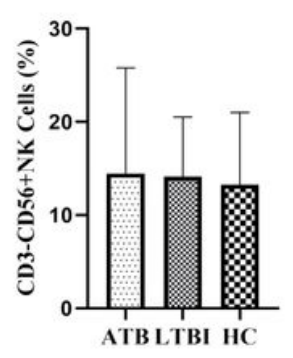

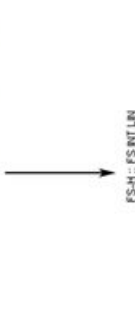

(c)

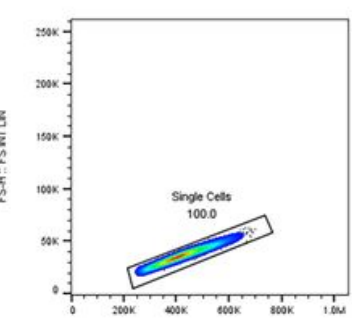

FS.A: FS NT LN

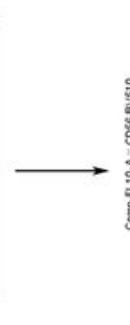

(d)

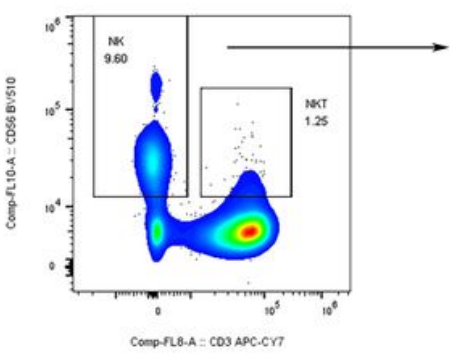

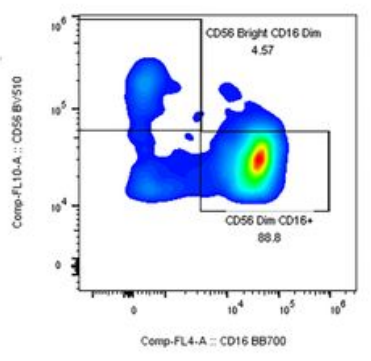

(e)

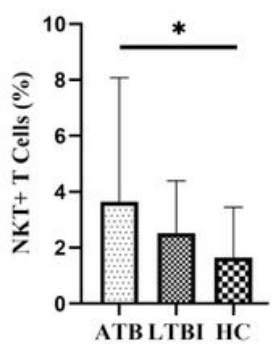

\section{Figure 1}

Gating strategies and the percentage of major NK cell subsets within study groups. a Gating strategies for NK subsets and NKT cells. $b$ The percentage of CD3-CD56+ NK cells from total lymphocytes in each of the three groups (ATB, $n=20 ; \mathrm{LTBI}, n=13 ; \mathrm{HC}, \mathrm{n}=14$ ). $c$-d The percentage of CD56DimCD16+ and CD56BrightCD16Dim NK cells from CD3-CD56+ NK in each of the three groups. e The percentage of 
CD3+CD 56+ NKT cells from total lymphocytes in each of the three groups. Results were expressed as the mean \pm SD. ${ }^{*} p<0.05,{ }^{*} p<0.01$.

(a)

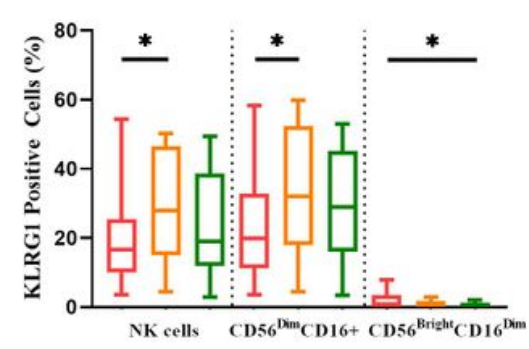

(b)

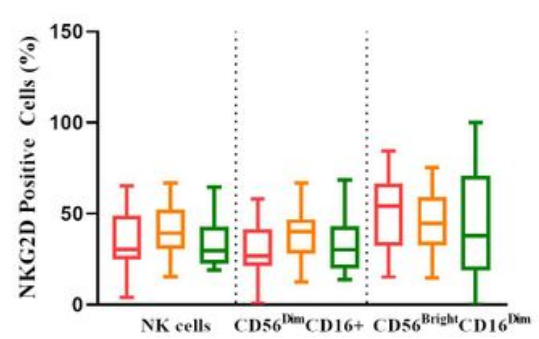

(c)

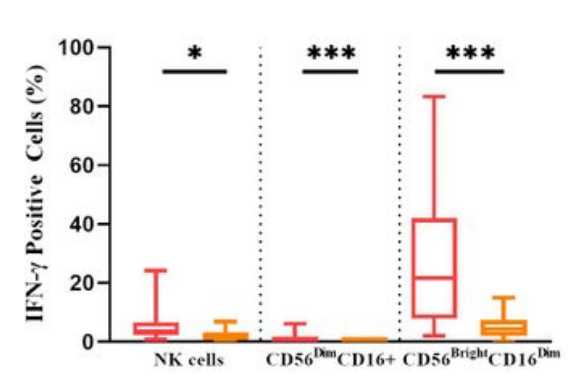

Figure 2

Surface receptors expression and IFN-y production of NK subsets from each of three participant groups. $a-b$ The expression of the inhibitory receptor KLRG1 and activating receptor NKG2D in NK subsets. c The production of IFN- $\gamma$ in NK subsets after Mtb specific antigen stimulation. Whiskers represented the maximum and minimum percentiles. ${ }^{\star} p<0.05,{ }^{* \star} p<0.01,{ }^{* \star *} p<0.001$. 
NK cells

(a)

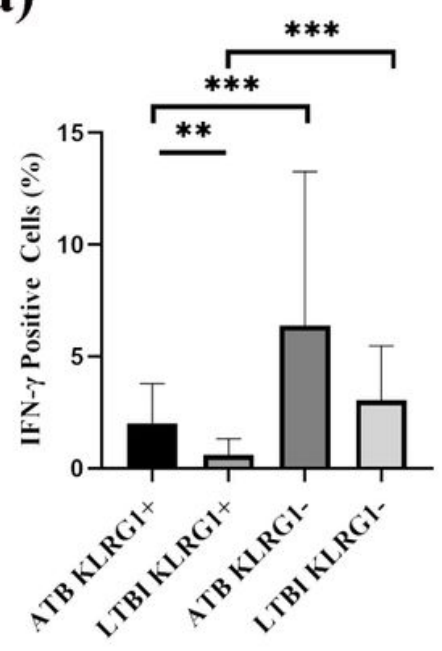

(b)

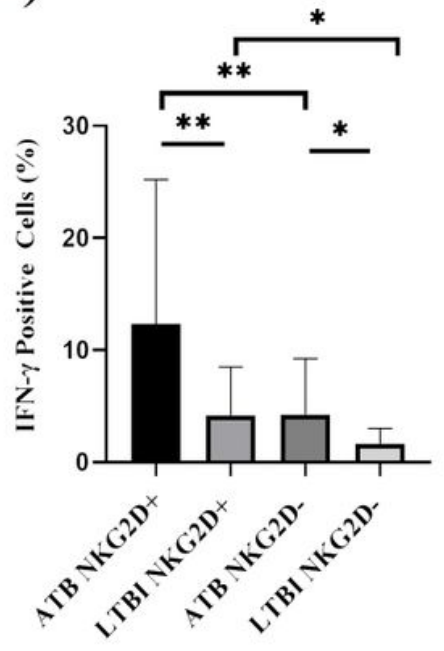

CD56 ${ }^{\mathrm{Dim}} \mathrm{CD} 16+\mathrm{NK}$

CD56 $^{\text {Bright }}{ }^{\text {CD16 }}{ }^{\text {Dim }}$ NK
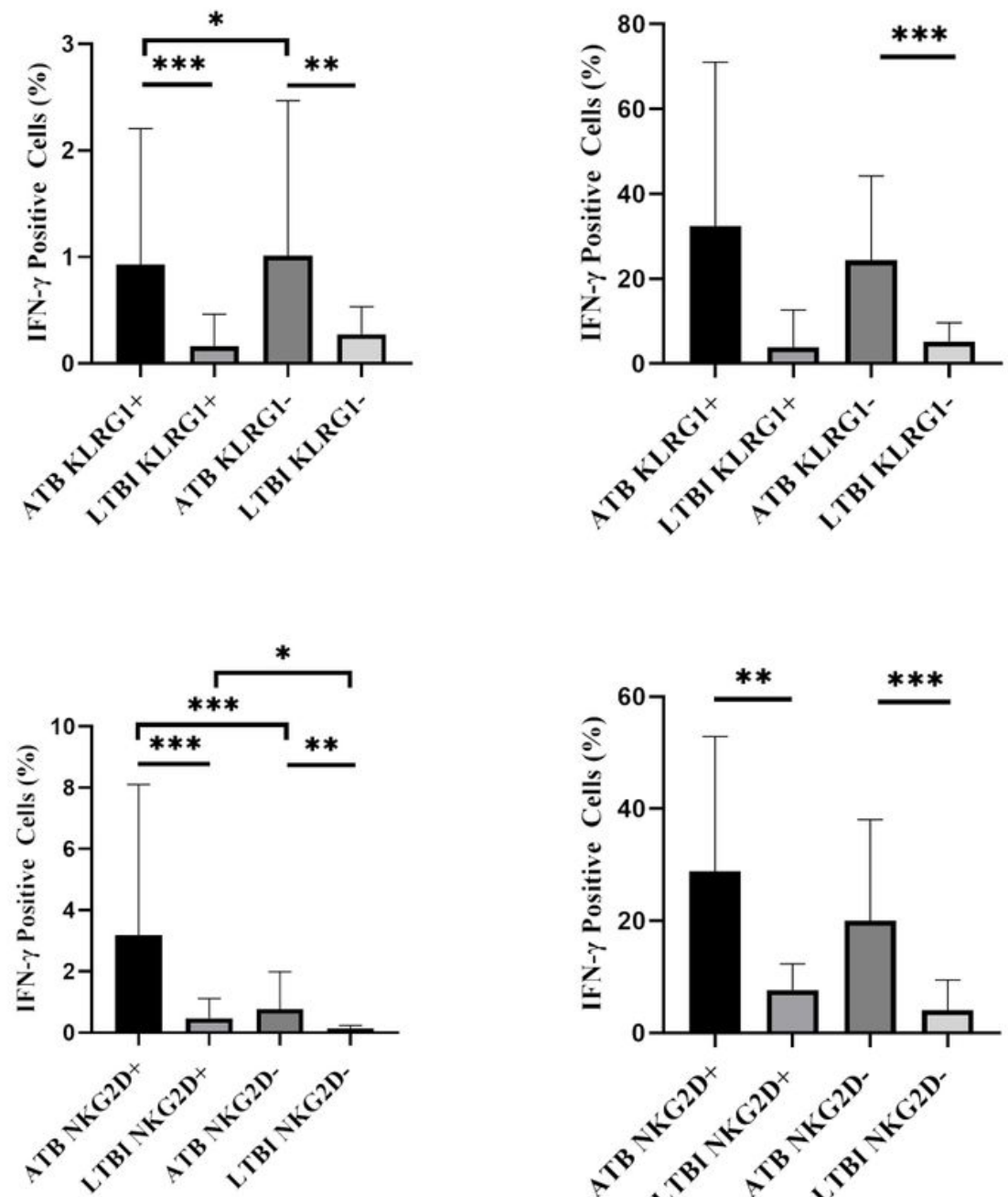

Figure 3

Cytokine secretion capacity in KLRG1+/- and NKG2D+/- NK subsets between ATB and LTBI. a Comparison the proportion of IFN-y between KLRG1+ and KLRG1 - NK subsets after Mtb specific antigen stimulation in TB-infected groups. b Comparison the proportion of IFN-y between NKG2D+ and NKG2DNK subsets after Mtb specific antigen stimulation in TB-infected groups. Results were expressed as the mean \pm SD. ${ }^{*} p<0.05,{ }^{* *} p<0.01,{ }^{* * *} p<0.001$. 
(a)

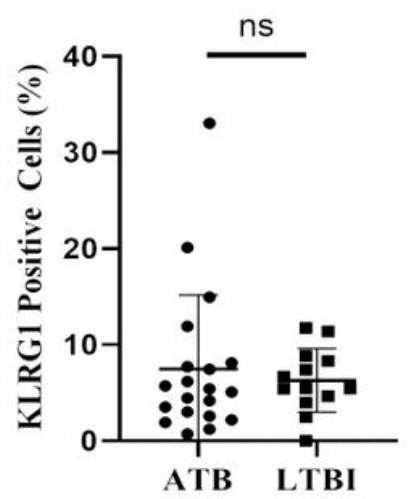

(b)

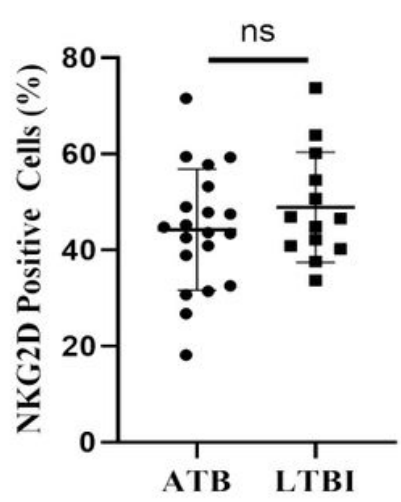

IFN- $\gamma^{+} \mathrm{CD56}^{\mathrm{Dim}} \mathrm{CD} 16+\mathrm{NK}$
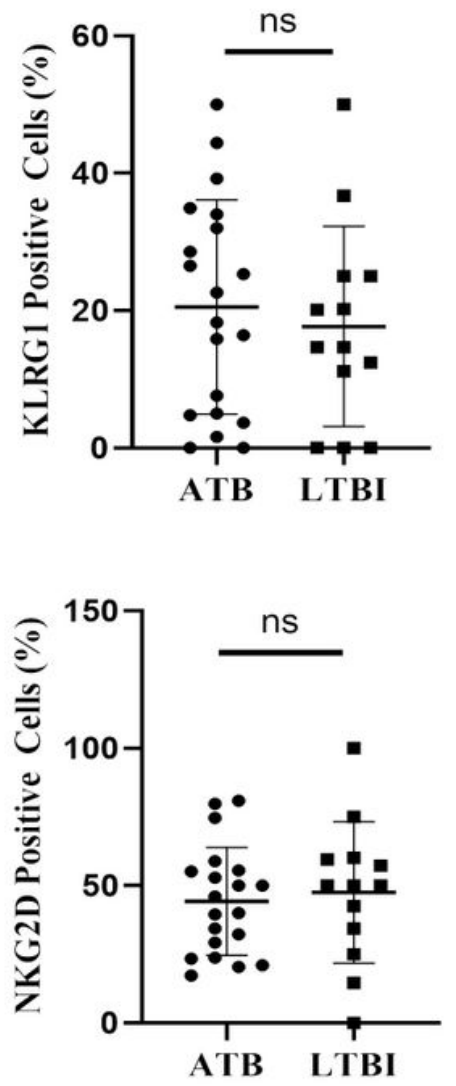

IFN- $\gamma^{+}$CD56 ${ }^{\text {Bright }}{ }^{\text {CD16 }}{ }^{\text {Dim }}$ NK
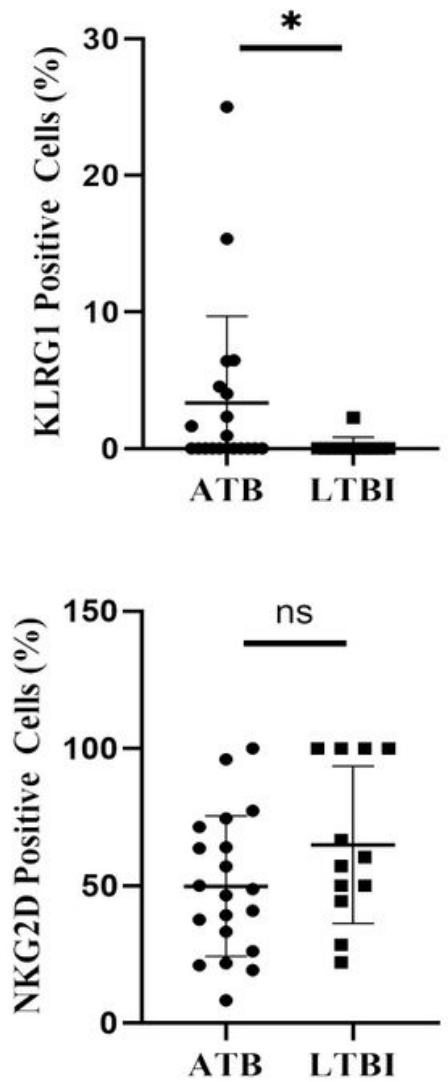

Figure 4

Expression of KLRG1 and NKG2D in Mtb specific IFN- $\gamma+$ NK subsets between ATB and LTBI. a The expression of KLRG1in IFN- -+ NK subsets after Mtb specific antigen stimulation in TB-infected groups. $b$ The expression of NKG2D in IFN- $\gamma+\mathrm{NK}$ subsets after Mtb specific antigen stimulation in TB-infected groups. ${ }^{*} p<0.05$. 
(a)

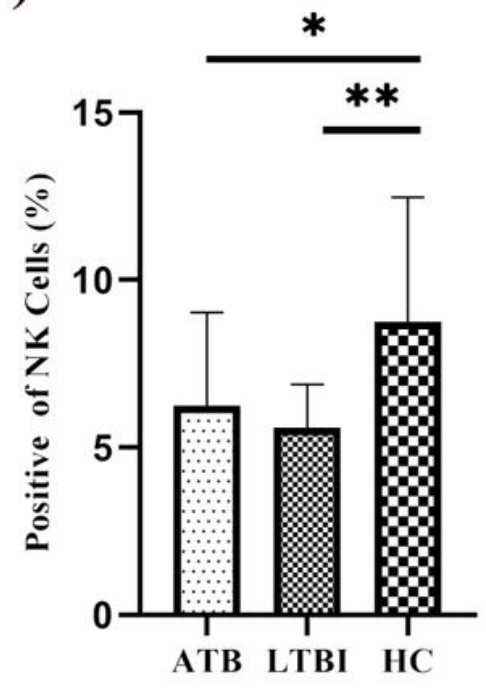

(b)

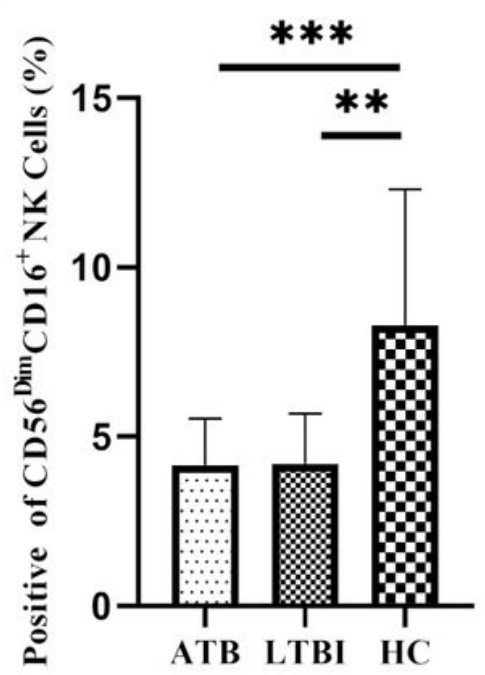

(c)

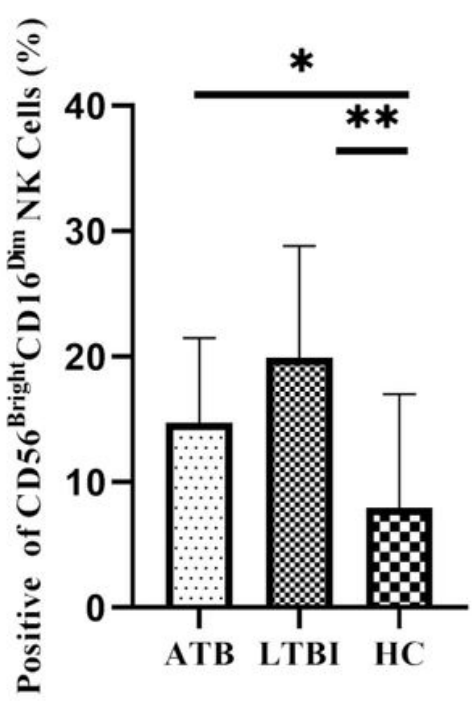

Figure 5

Expression of NK subsets defined by CD27 in the three participant cohorts. a The percentage of CD27 in total NK cells (CD3-CD56+). b-c The expression of CD27 in CD56 and CD16 defined NK subsets (CD56DimCD16+ and CD56BrightCD16Dim). Results were expressed as the mean $\pm S D$. ${ }^{*} p<0.05$, ${ }^{*} p<$ $0.01, * \star * p<0.001$.

(a)

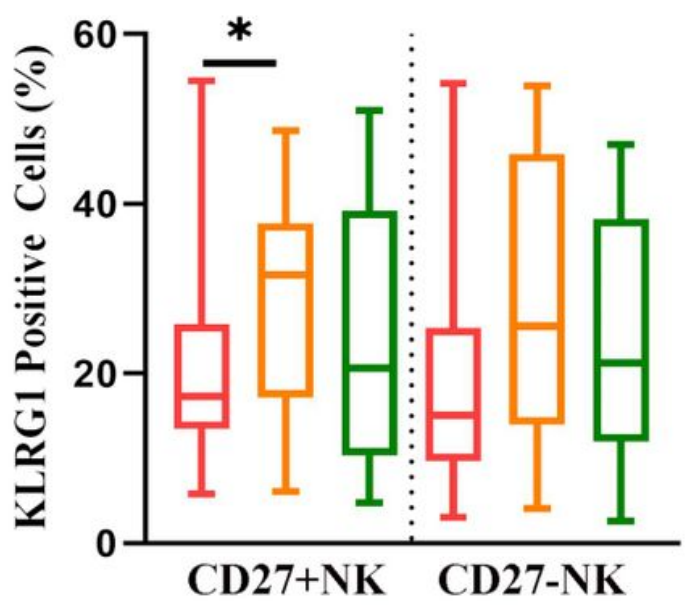

(b)

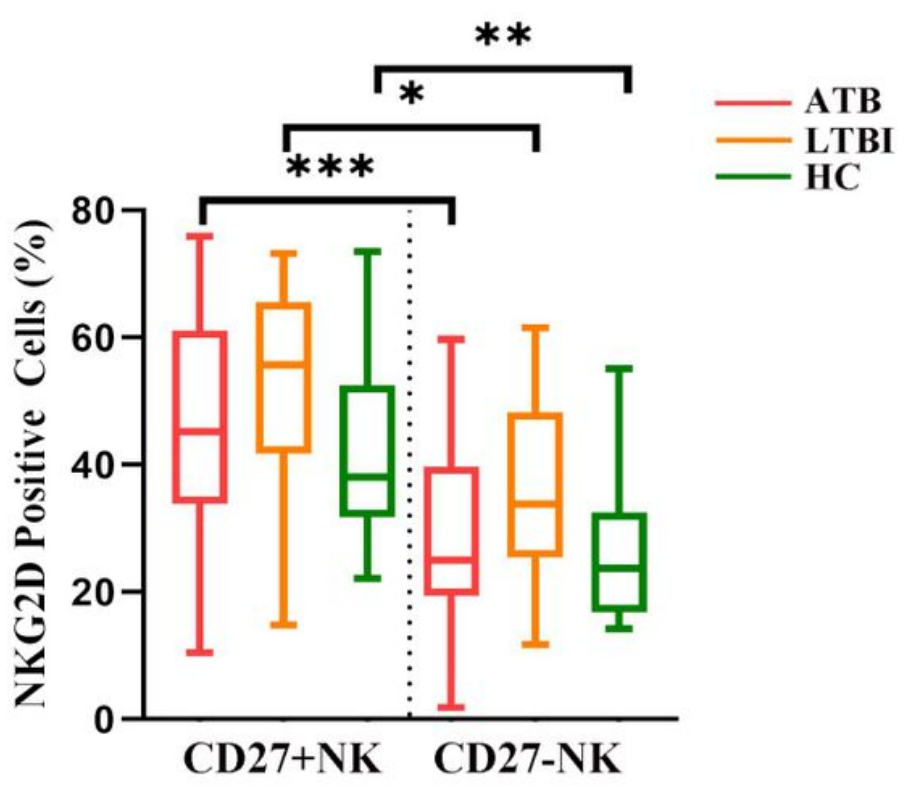

Figure 6

Expression of NK surface receptors between CD27+ and CD27- NK subsets. a Comparison the proportion of KLRG1 between CD27+ and CD27- NK subsets in three participant groups. b Comparison the 
proportion of NKG2D between CD27+ and CD27- NK subsets in the three participant groups. Whiskers represented the maximum and minimum percentiles. ${ }^{\star} p<0.05,{ }^{\star \star} p<0.01,{ }^{\star \star \star} p<0.001$.

\section{Supplementary Files}

This is a list of supplementary files associated with this preprint. Click to download.

- Additionalfile1.pdf

- Additionalfile2.pdf

- Additionalfile3.pdf 